\title{
Millimeter Wave Propagation through Plasma
}

\author{
S. K. Chakrabarti ${ }^{1}$, J. Akhter ${ }^{1}$ and R. Sinha ${ }^{2}$ \\ ${ }^{1}$ Tribhuvan University, P.G. Dept. of Physics, M. M. A. M. Campus, Biratnagar \\ ${ }^{2}$ B. N. Mandal University, Central Dept. of Physics, Bihar, India \\ Corresponding author: skc_2007@yahoo.com
}

\begin{abstract}
:
Theoretical investigation has been carried out to evaluate the attenuation constant and phase velocity of millimetre wave in plasma. For this purpose the expression for the permittivity of plasma medium is obtained. The concept of Faraday rotation has been introduced for utilisation of the same for further calculation. It has been found that attenuation constant and phase velocity both increase with increasing value of plasma frequency. However, the value of attenuation constant decreases with increasing operational frequency.
\end{abstract}

Key words : plasma, millimetre wave, faraday effect, attenuation constant, phase velocity.

\section{Introduction}

Recently considerable interest has been developed to evaluate the effect of plasma on the millimetre wave propagation. The phase and amplitude of the wave are affected and cause attenuation. Here attempt has been made to establish the expressions for attenuation constant and phase velocity in terms of plasma parameters.

\section{Theoretical consideration}

In order to evaluate the effect of plasma medium on millimetre wave propagation, Maxwell's equation and the equation of motion of electron have been utilised. Since the oscillation in plasma is harmonic, so the variations in electron density and velocity of electrons will also be of harmonic nature. Thus for small sinusoidal oscillation the effective time $(\mathrm{t})$ varying electron density $(\eta)$ and electron velocity (v) can be written as

$$
\eta=\mathrm{N}+\operatorname{Re}\left\{\mathrm{ne}^{\mathrm{j} \omega \mathrm{t}}\right\} \quad[\mathrm{j}=\sqrt{-1}]
$$

and $\quad \mathrm{v}=\operatorname{Re}\left\{\mathrm{ve}^{\mathrm{j} \omega \mathrm{t}}\right\}, \quad[\omega=$ operational frequency]

where $\mathrm{N}$ is the steady electron density and $v$ the frequency of oscillation.

Now, the time varying electron current density can be expressed as

$$
\mathrm{J}=\eta \mathrm{ev}
$$

Putting into equation (iii) the values of $\eta$ and ' $v$ ' from equations (i) and (ii) respectively, one has

$$
\begin{aligned}
& \mathrm{J}=\left[\mathrm{N}+\operatorname{Re}\left\{\mathrm{ne}^{\mathrm{j} \omega \mathrm{t}}\right\}\right] \text { e } \operatorname{Re}\left\{v \mathrm{e}^{\mathrm{j} \omega t}\right\} \\
& =\operatorname{Ne} \operatorname{Re}\left\{v \mathrm{e}^{\mathrm{j} \omega \mathrm{t}}\right\}+\mathrm{e} \operatorname{Re}\left\{\mathrm{ne}^{\mathrm{j} \omega \mathrm{t}}\right\} \operatorname{Re}\left\{v \mathrm{e}^{\mathrm{j} \omega \mathrm{t}}\right\} \\
& =\operatorname{Ne} \operatorname{Re}\left\{v \mathrm{e}^{\mathrm{j} \omega t}\right\} \\
& \text { Using Maxwell's equation in phasor form : } \\
& \quad \nabla \times \mathrm{H}=\mathrm{j} \omega \varepsilon_{\mathrm{v}} \mathrm{E}+\mathrm{J}
\end{aligned}
$$

and the equation of motion of electron in time varying form and also noting that

$$
\mathrm{J}=\sigma \mathrm{E}, \quad[\sigma=\text { dyadic }]
$$

the permittivity $\left(\varepsilon_{v}\right)$ of the plasma may be obtained as $^{7}$

$$
\begin{aligned}
& {\left[\begin{array}{lll}
0 & \frac{\partial}{\partial z} & \frac{\partial}{\partial y} \\
-\frac{\partial}{\partial y} & 0 & \frac{\partial}{\partial x} \\
-\frac{\partial}{\partial y} & \frac{\partial}{\partial x} & 0
\end{array}\right]\left[\begin{array}{l}
H_{x} \\
H_{y} \\
H_{z}
\end{array}\right]=j \omega \varepsilon_{v}\left[\begin{array}{lll}
K^{\prime} & K^{\prime \prime} & 0 \\
-K^{\prime} & K^{\prime} & 0 \\
0 & 0 & K_{0}
\end{array}\right]\left[\begin{array}{l}
E_{x} \\
E_{y} \\
E_{z}
\end{array}\right]} \\
& \text { where } \quad \mathrm{K}_{0}=1-\frac{\chi}{\mathrm{U}}, \quad\left[\chi=\frac{\omega_{\mathrm{p}}^{2}}{\omega^{2}}\right] \\
& \mathrm{K}^{\prime}=1-\frac{\mathrm{U} \chi}{\mathrm{U}^{2}-\gamma^{2}} \quad[\mathrm{U}=1-\mathrm{jz}] \\
& \text { and } \quad \mathrm{K} "=1-\frac{\chi}{\mathrm{U}^{2}-\gamma^{2}}, \quad\left[\gamma=\frac{\omega_{\mathrm{c}}}{\omega}\right]
\end{aligned}
$$

$\omega_{\mathrm{c}}$ and $\omega_{\mathrm{p}}$ being the cyclotron and plasma frequencies respectively.

Now, any linearly polarised wave may be regarded as the vector sum of two counter-rotating circularly polarised waves. If such a wave propagates in the direction of the magnetic field in a lossless plasma, the two circularly polarised components 
will travel at different phase velocities and the plane of polarisation will rotate with distance. The phenomenon, known as Faraday effect, is exhibited by a plane-polarised millimetre wave passing through ionosphere. The propagation of two equalmagnitude circularly polarised waves in a lossless plasma in the direction of $\mathrm{z}$-axis may be represented by the electric field in the form ${ }^{5}$

$$
\mathrm{E}=\mathrm{a}_{\mathrm{r}} \mathrm{e}^{-\mathrm{j} \beta_{\mathrm{r}} \mathrm{z}}+\mathrm{a}_{\mathrm{l}} \mathrm{e}^{-\mathrm{j} \beta_{\mathrm{l}} \mathrm{z}},
$$

where $a_{r} \& a_{1}$ are unit vectors and $\beta_{r} \& \beta_{1}$ the propagation factors for right and left circularly polarised waves respectively.

Therefore, rotation in the resultant $\mathrm{E}$ vector is given by ${ }^{4}$

$$
\begin{aligned}
\Omega & =\frac{1}{2}\left(\beta_{\mathrm{r}}-\beta_{1}\right) \mathrm{z} \\
& =\frac{\grave{\mathrm{u}}}{2 \mathrm{c}}\left(\mathrm{n}_{1}-\mathrm{n}_{\mathrm{r}}\right) \mathrm{z},
\end{aligned}
$$

where ' $c$ ' is the absolute velocity of light and $n_{1} \& n_{r}$ the refractive indices for the left and right circularly polarised waves respectively.

If the signal frequency is higher as compared to the plasma and cyclotron frequencies, the angle of Faraday rotation can be derived as ${ }^{1}$

$$
\Omega=\frac{\mathrm{e}^{3} \mathrm{~B}_{0}}{2 \mathrm{~cm}^{2} \varepsilon_{v} \omega^{2}} \mathrm{Nz}
$$

where ' $\mathrm{e}$ ' and ' $\mathrm{m}$ ' are the charge and mass of electron respectively. Thus, the rotation of the plane of polarisation is not only proportional to the distance travelled but also to the electron density.

If the electron density in plasma varies with distance and if $\mathrm{B}_{0}$ is not constant i.e. the magnetic field (B) is changing in plasma in the direction of wave propagation, then equation (iv) takes the form $^{2}$

$$
\Omega=\frac{\mathrm{e}^{3}}{2 \mathrm{~cm}^{2} \varepsilon_{v} \omega^{2}} \int \mathrm{B} \cos \theta \mathrm{N} \text { ds }
$$

where $\theta$ is the angle between the ray and the magnetic field and ds an elementary surface in the direction of the ray.

In case of millimetre waves travelling in ionosphere under the influence of earth's magnetic field, equation (v) is transformed as ${ }^{6}$
$\Omega=\frac{\mathrm{e}^{3}}{8 \pi^{2} \mathrm{~m}^{2} \varepsilon_{v} \mathrm{f}^{2}} \int_{0}^{\mathrm{h}_{\mathrm{t}}} \mathrm{B} \cos \theta \cos \mathrm{e}(\delta+\tau) \mathrm{Ndh}$,

where $h_{t}$ is the height upto which the ionosphere exists, $\delta$ the total separation angle, $\tau$ the elevation of ray at the earth station and dh the elementary increase in altitude perpendicular to the earth surface. Putting the known values of e, $\mathrm{m}$ and $\varepsilon_{v}$ in equation (vi), one gets

$$
\Omega=\frac{2.365 \times 10^{4}}{\mathrm{f}^{2}} \int_{0}^{\mathrm{h}_{\mathrm{t}}} \phi(\mathrm{h}, \mathrm{T}) \mathrm{N}(\mathrm{h}) \mathrm{dh}
$$

Defining a function $\phi(\mathrm{h}, \mathrm{T})$ as

$$
\phi(\mathrm{h}, \mathrm{T})=\mathrm{B} \cos \theta \operatorname{cosec}(\delta+\tau),
$$

equation (vii) becomes

$$
\Omega=\frac{2.365 \times 10^{4}}{\mathrm{f}^{2}} \int_{0}^{\mathrm{h}_{\mathrm{t}}} \mathrm{B} \cos \theta \cos \mathrm{e}(\delta+\tau) \mathrm{N} \mathrm{dh}
$$

The height of the ionosphere is normally assumed to be around $340-400 \mathrm{~km}$., which is near the centre of distribution of electron concentration. Thus, if $\phi(\mathrm{h}, \mathrm{T})$ is calculated at a mean ionosphere height $h_{m}$, then it can be assumed constant having the value $\phi\left(\mathrm{h}_{\mathrm{m}}, \mathrm{T}\right)$ throughout the path of the ray. So, equation (viii) is transformed to

$$
\Omega=\frac{2.365 \times 10^{4}}{\mathrm{f}^{2}} \phi\left(\mathrm{h}_{\mathrm{m}}, \mathrm{T}\right) \int_{0}^{\mathrm{h}_{\mathrm{t}}} \mathrm{N}(\mathrm{h}) \mathrm{dh}
$$

Also $\gamma$ can be expressed as

$$
\gamma=\alpha+\mathrm{j} \beta
$$

Separating the real and imaginary parts in equations (viii) and (ix) one has ${ }^{3}$

$$
\begin{aligned}
\alpha & =\omega \sqrt{\mu \varepsilon} \frac{\sigma}{2 \omega \varepsilon} \\
& =\frac{\sigma}{2} \sqrt{\frac{\mu}{\varepsilon}}
\end{aligned}
$$

and $\beta=\omega \sqrt{\mu}\left(1+\frac{1}{8} \frac{\sigma^{2}}{\omega^{2} \varepsilon^{2}}\right)$

The values of attenuation constant $(\alpha)$ and complex phase propagation constant $(\beta)$ can be modified for plasma as follows :

and

$$
\begin{gathered}
\alpha=\frac{\sigma}{2} \sqrt{\frac{\mu_{0}}{\varepsilon_{\mathrm{p}}}} \\
\beta=\omega \sqrt{\mu_{0} \varepsilon_{\mathrm{p}}}\left(1+\frac{1}{8} \frac{\sigma^{2}}{\omega^{2} \varepsilon_{\mathrm{p}}^{2}}\right)
\end{gathered}
$$


Putting the value of $\varepsilon_{\mathrm{p}}$ one has

$$
\alpha=\frac{\sigma}{2} \sqrt{\frac{\mu_{0}}{\varepsilon_{0}\left(1-\frac{\omega_{\mathrm{p}}^{2}}{\omega^{2}}\right)}}
$$

and

$$
\sqrt{\mu_{0} \varepsilon_{0}\left(1-\frac{\omega_{\mathrm{p}}^{2}}{\omega^{2}}\right)}\left[1+\frac{1}{8} \frac{\sigma^{2}}{\omega^{2} \varepsilon_{0}^{2}}\left(1-\frac{\omega_{\mathrm{p}}^{2}}{\omega^{2}}\right)^{-2}\right] \beta=\omega
$$

Now, the phase velocity of the wave in plasma medium is given by

$$
\begin{aligned}
& \mathrm{v}_{\mathrm{ph}}=\frac{\omega}{\beta} \\
& =\frac{1}{\sqrt{\mu_{0} \varepsilon_{0}\left(1-\frac{\omega_{\mathrm{p}}^{2}}{\omega^{2}}\right)}\left[1+\frac{1}{8} \frac{\sigma^{2}}{\omega^{2} \varepsilon_{0}^{2}}\left(1-\frac{\omega_{\mathrm{p}}^{2}}{\omega^{2}}\right)^{-2}\right]}
\end{aligned}
$$

\section{Numerical computation}

Computational work has been done using equations (x) and (xi). The data thus obtained are shown in the form of graphs vide Fig. -1 and Fig.-2.

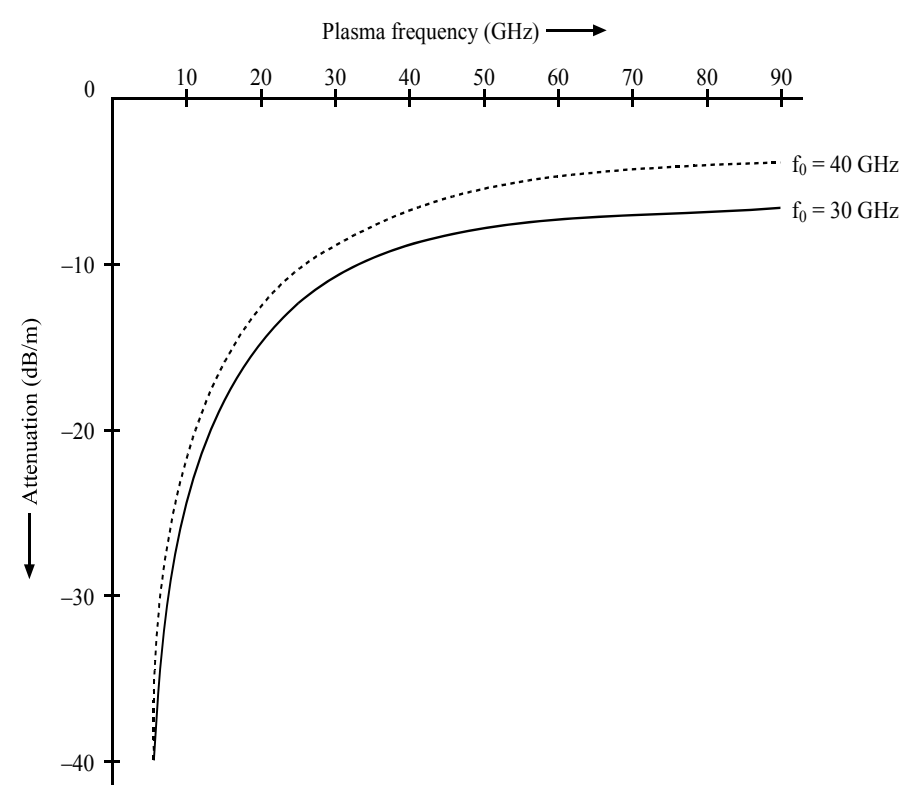

Fig.-1 : Variation of attenuation with plasma frequency

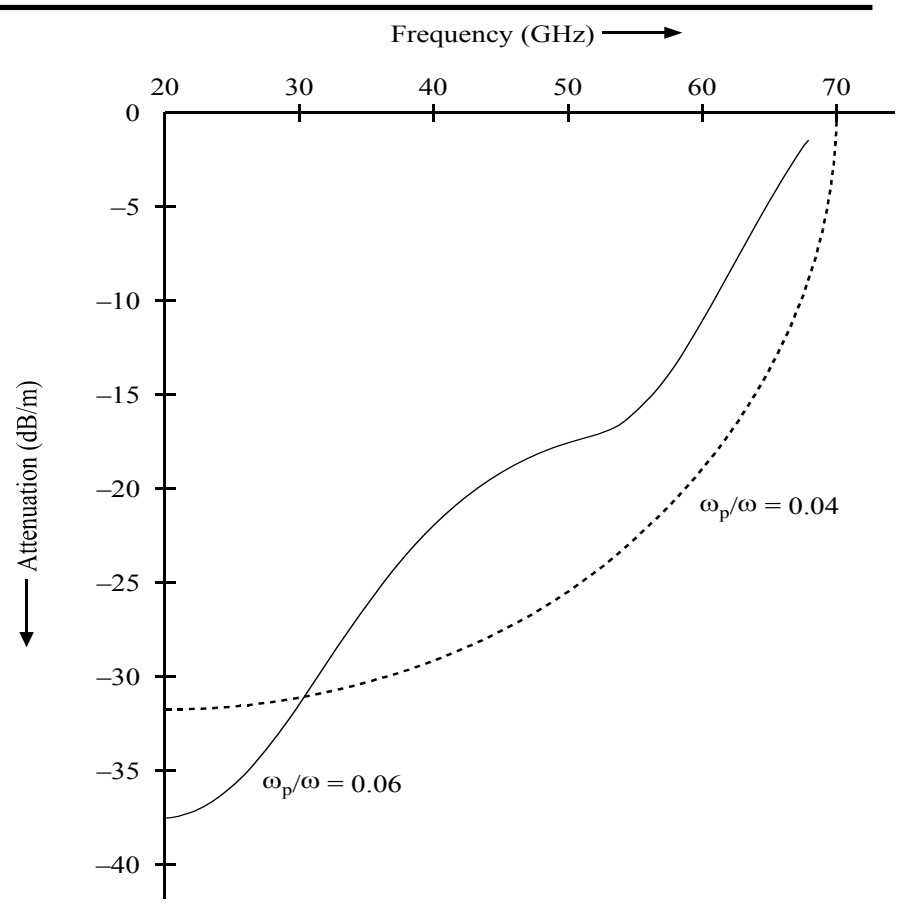

Fig. -2 : Variation of attenuation

\section{Results and discussion}

Using equations (x) and (xi) the values of attenuation constant and phase velocity have been obtained. It has been found from Fig. -1 that the attenuation constant increases with increasing plasma frequency. However, it decreases with increasing operational frequency. The value of phase velocity increases with plasma frequency as well as operational frequency. This is corroborated with the fact that the increase in operational frequency makes the electrons oscillate giving rise to radiation in all directions and thereby causing decrease in attenuation of millimetre waves in plasma medium.

\section{References}

1. Bunkin, F., Soviet JETP, 5 (1957) 277

2. Galeis, J., Terrestrial propagation of low electromagnetic waves, Pergamon Press, Oxford, 1972

3. Ishimaru, A., Wave propagation and scattering in random media, Academic, New York, 1978

4. Kerr, D., Propagation of short radio wave, McGraw Hill Publication, New York, 1951

5. Sankaran, V., Schronenberger, K. and Walsh, J. T., App. Opt., 38 (1999) 4252

6. Singh, S., Rai, C. S. and Vishvakarma, B. R., J. Inst. Engrs. Ind., 75 (1994) 37

7. Wait, J. R., Electromagnetic waves in stratified media, Pergamon Press, Oxford, 1962

\section{वण}

